\title{
Ruxolitinib for the treatment of patients with steroid-refractory GVHD: an introduction to the REACH trials
}

\author{
Madan Jagasia*,1, Robert Zeiser², Michael Arbushites ${ }^{3}$, Patricia Delaite $^{3}$, Brian Gadbaw ${ }^{4}$ \& \\ Nikolas von Bubnoff ${ }^{2}$ \\ ${ }^{1}$ Vanderbilt-Ingram Cancer Center, 1301 Medical Center Dr \#1710, Nashville, TN, USA 37232 \\ ${ }^{2}$ Department of Hematology, Oncology \& Stem Cell Transplantation, University Hospital Freiburg, Hugstetter Str. 55, 79106 \\ Freiburg im Breisgau, Germany \\ ${ }^{3}$ Incyte Corporation, 1801 Augustine Cut-off, Wilmington, DE, USA 19803 \\ ${ }^{4}$ Novartis Pharmaceuticals Corporation, 1 Health Plaza, East Hanover, NJ, USA 07936 \\ * Author for correspondence: Tel.: +1 615936 8422; Fax: +1 615936 1812; madan.jagasia@Vanderbilt.edu
}

For patients with hematologic malignancies and disorders, allogeneic hematopoietic stem cell transplantation offers a potentially curative treatment option. Many patients develop graft-versus-host disease (GVHD), a serious complication and leading cause of nonrelapse mortality. Corticosteroids are the standard first-line treatment for GVHD; however, patients often become steroid-refractory or remain corticosteroiddependent. New second-line treatment options are needed to improve patient outcomes. Here we review the role of JAK1 and JAK2 in acute and chronic GVHD. We also describe the study designs of the Phase II REACH1 (NCT02953678) and the Phase III REACH2 (NCT02913261) and REACH3 (NCT03112603) clinical trials that are currently recruiting patients to evaluate the JAK1/JAK2 inhibitor ruxolitinib in patients with corticosteroid-refractory acute or chronic GVHD.

First draft submitted: 30 October 2017; Accepted for publication: 18 December 2017; Published online: 9 January 2018

Keywords: allogeneic stem cell transplant $\bullet$ JAK $\bullet$ refractory graft-versus-host disease

Allogeneic hematopoietic stem cell transplantation (HSCT) is an immunotherapeutic approach that provides the only potentially curative option for patients with high-risk or relapsed hematologic malignancies, including leukemia, myelodysplastic syndrome, lymphoma and myeloma [1-3], and is also used to treat several nonmalignant hematologic conditions and inherited metabolic disorders [3,4]. HSCT clinical benefit is in part a result of the graft-versus-leukemia (GVL) effect, in which a donor immune response is targeted against recipient malignant cells [5]. Although alloreactive donor T cells play an important role in GVL by targeting tumor cells for elimination, the serious complication graft-versus-host disease (GVHD) develops when alloreactive donor cells attack healthy host tissues [5]. Despite promising advances in HSCT methodology, including prophylactic immunosuppressive therapies, approximately $50 \%$ of HSCT recipients develop GVHD [6].

GVHD can present as an acute (aGVHD) or a chronic (cGVHD) disease $[7,8]$. Both aGVHD and cGVHD are inflammatory disorders initiated by the infiltration of alloreactive $T$ cells into target organs [9], followed by activation of proinflammatory signaling cascades [10], tissue damage and organ failure [8]. Previously, the distinction between aGVHD and cGVHD was based solely on the time of onset (i.e., 100 days post-transplant). However, important pathophysiological distinctions have since been identified, requiring evaluation of clinical presentation to make an accurate disease diagnosis [11]. The skin is the organ most typically affected at the onset of aGVHD, followed by the GI tract and liver [7]. Several organ systems, including the skin and GI tract, are also affected in cGVHD, but clinical distinctions can be made to differentiate cGVHD from aGVHD in these organ systems [11]. Additional diagnostic symptoms of cGVHD manifest in the mouth, genitalia, lungs and muscles [11]. The target organ damage observed in aGVHD is primarily characterized by apoptosis, whereas CGVHD is associated with fibrosis and many autoimmune features, indicative of an expanded role for macrophages and B cells compared with aGVHD [12,13]. 
GVHD risk depends on several factors, including donor-recipient human leukocyte antigen (HLA) disparity, patient age, the source of the donor cells, conditioning regimen and the type of post-transplant GVHD prophylaxis [14,15]. The risk factors for development of GVHD are similar for acute and chronic disease, and a diagnosis of aGVHD is itself a significant predictor of cGVHD development [14]. The incidence of grade 2-4 aGVHD ranges from 26 to 59\% within the first 100 days after transplant [11,14]. Even among HLA-identical donor-recipient pairs, aGVHD occurs in 39\% of patients receiving grafts from matched sibling donors and $59 \%$ receiving unrelated donor (URD) cells [11]. In cases of 1-mismatch URD grafts, aGVHD occurs in $60-80 \%$ of patients and is associated with decreased survival $[16,17]$. Multiple mismatches are associated with significantly worse survival and increased rates of grade $3 / 4$ aGVHD [17]. Among HLA-matched donor-recipient pairs, the incidence of cGVHD within 2 years post-transplant is estimated to occur in $7-40 \%$ of patients [14]. A retrospective study of nearly 3000 patients who received HSCT from related or unrelated donors under systemic immunosuppression revealed a 2 year cumulative cGVHD incidence of 34\% [15].

GVHD is a leading cause of nonrelapse mortality (NRM) following both HLA-matched sibling donor and URD transplantation $[8,18,19]$. Data from a large retrospective cohort study found that cumulative incidences of mortality following transplants using matched sibling donor cells $(\mathrm{n}=3191)$ were 21,28 and $31 \%$ at 1,3 and 5 years, respectively [11]. Among patients receiving URD transplants $(\mathrm{n}=2370)$, the cumulative incidences of transplant-related mortality were 31,37 and $40 \%$ at 1,3 , and 5 years, respectively [11].

Systemic corticosteroids are the standard of care for the initial treatment of grade $2-4$ aGVHD [20,21] and cGVHD [21,22]. However, many patients with aGVHD or cGVHD do not experience sustained responses to corticosteroids [1,23], and 6 month survival rates among steroid-refractory (SR) patients are approximately $49 \%$ with long-term survival rates of only $5-30 \%[20,24]$. The Bruton tyrosine kinase inhibitor ibrutinib recently received US FDA approval for the treatment of cGVHD after failure of $\geq 1$ treatment based on results from a single-arm Phase Ib/II study in which patients with cGVHD and $\geq 1$ prior treatment regimen failure achieved an average overall response rate (ORR) of $67 \%$ (21\% complete response [CR]; median follow-up, 13.9 months following start of ibrutinib administration) [25]. There are currently no FDA-approved medications for SR aGVHD, and there is a lack of well controlled Phase II/III clinical trial results to support the use of alternative therapies in both aGVHD and cGVHD [20]. Indeed, current recommendations encourage enrollment in clinical trials for patients with GVHD following first-line failure of steroid therapy [21]. Here we briefly review the rationale for treating patients with aGVHD or CGVHD with the JAK1/JAK2 inhibitor ruxolitinib and provide an overview of the study designs of three prospective clinical trials evaluating ruxolitinib in GVHD settings (Ruxolitinib in Patients With Refractory GVHD After Allogeneic Stem Cell Transplantation [REACH] 1-3).

\section{Overview of ruxolitinib \& rationale for targeting JAK signaling for SR GVHD}

The JAKs are intracellular nonreceptor tyrosine kinases that are promising targets for GVHD treatment and prophylaxis given their critical role in cytokine signaling and the development and function of several immune cell types [26]. Preclinical and clinical evidence support a role for JAK1 and JAK2 signaling in the pathogenesis of GVHD. In dendritic cell (DC) culture experiments, inhibition of JAK2 signaling with TG101348 significantly suppressed T-cell proliferation and activation and promoted a phenotypic shift favoring $\mathrm{CD}^{+}{ }^{+}$regulatory $\mathrm{T}$ cells over $\mathrm{CD}^{+} \mathrm{CD} 25^{+}$effector $\mathrm{T}$ cells in mixed lymphocyte reaction assays [27]. Inhibition of IL-6, a cytokine whose effects are mediated via JAK1/JAK2 signaling [26], increased the number of regulatory $\mathrm{T}$ cells in a murine model of GVHD in the colon, liver and lung, leading to an attenuation of disease severity [28]. In agreement with these effects, the frequency of regulatory $\mathrm{T}$ cells is reduced in allogeneic HSCT patients with cGVHD versus those without cGVHD and healthy controls [29]. Preclinical data also support a role for JAK signaling in B-cell development and activation. In an in vitro study of human B cell cultures, ruxolitinib completely blocked IFN- $\gamma$ mediated expansion of $\mathrm{CD} 38^{+} \mathrm{CD} 27^{-}$germinal center $\mathrm{B}$ cells, while the JAK1/3 inhibitor tofacitinib partially inhibited B-cell expansion [30], suggesting that JAK signaling could play a role in the pathogenesis of B-cell-mediated cGVHD.

Ruxolitinib is an oral selective JAK1/JAK2 inhibitor with equipotent activity toward JAK1 and JAK2 (IC 50 values of 3.3 and $2.8 \mathrm{nM}$, respectively) [31]. Ruxolitinib has been approved by the FDA for the treatment of intermediate or high-risk myelofibrosis, including primary myelofibrosis, postpolycythemia vera myelofibrosis and postessential thrombocythemia myelofibrosis, and patients with polycythemia vera who have had an inadequate response to or are intolerant of hydroxyurea [32]. 
Preclinical studies demonstrated promising anti-GVHD activity with ruxolitinib. In vitro and in vivo analyses have shown that ruxolitinib affects several aspects of immune function related to GVHD, including inhibiting antigen-presenting cell function through inhibition of DC differentiation, reducing DC migratory capacity [33], decreasing DC expression of major histocompatibility complex class II [34], reducing T-cell proliferation and activation and decreasing cytokine production [35]. Furthermore, ruxolitinib treatment in murine models of GVHD was associated with the amelioration of disease manifestations and symptoms. In murine models of aGVHD, mice receiving ruxolitinib had lower grade aGVHD in the skin, liver and intestines [9], improved survival, reduced TNF- $\alpha$ and IL-12 levels, reduced levels of allogeneic and central memory T cells and increased levels of regulatory $T$ cells in the spleen and GI tract [35]. Topical ruxolitinib treatment in a murine model of skin GVHD was associated with less severe skin GVHD scores, reduced T-cell infiltration of the skin and maintenance of LGR5+ hair follicle stem cell levels that are normally reduced with the onset of GVHD [36]. Importantly, data from murine models suggest that ruxolitinib does not inhibit GVL activity. A strong GVL effect of alloreactive donor T cells was maintained after ruxolitinib administration [9]. In murine dual models for leukemia or lymphoma relapse and GVHD, ruxolitinib was associated with improvements in survival and GVHD in gastrointestinal organs, liver and skin, without affecting GVL activity $[37,38]$.

Several retrospective clinical studies have evaluated ruxolitinib treatment in patients with SR aGVHD or cGVHD. A retrospective survey from 19 stem cell transplant centers in Europe and the USA evaluated outcomes of 95 patients with SR GVHD who received ruxolitinib as salvage therapy [39]. In patients with aGVHD, the ORR was $81.5 \%$ (45/54), including a CR rate of $46.3 \%$ (25 patients). In patients with cGVHD, the ORR was $85.4 \%$ (35/41), including 32 patients (78.0\%) with partial response (PR) and three patients (7.3\%) with CR. The 6 month survival estimate and GVHD relapse rates were 79 (95\% CI: 67.3-90.7\%) and 6.8\%, respectively, for patients with aGVHD and 97.4 (95\% CI: 92.3-100\%) and 5.7\%, respectively, for patients with cGVHD [39]. Furthermore, low disease recurrence rates of the underlying malignancy were observed $(9.3$ and $2.4 \%$ in patients with aGVHD and cGVHD, respectively), suggesting that ruxolitinib treatment did not impair the GVL effect [39]. Clinical improvements with ruxolitinib treatment were observed in patients with aGVHD in GI organs, liver or skin, and in patients with cGVHD in skin, liver, GI tract, lungs or musculoskeletal tissue. Long-term follow-up (median: aGVHD, 19 months; cGVHD, 24 months) revealed that $41 \%$ of patients with aGVHD and $24 \%$ with cGVHD had an ongoing response and were free of immunosuppression [40]. One-year overall survival (OS) rates were 62.4 (95\% CI: 49.4-75.4\%) and 92.7\% (95\% CI: 84.7-100\%) for SR aGVHD and cGVHD, respectively [40]. Adverse events (AEs) were primarily cytomegalovirus reactivation (aGVHD: 33\%; cGVHD: 15\%), grade 3/4 cytopenia (33\%; 7\%), and grade $1 / 2$ cytopenia $(22 \% ; 10 \%)$. A study of four patients treated with ruxolitinib for aGVHD $(\mathrm{n}=1)$ or cGVHD $(\mathrm{n}=3)$ following HSCT for myelofibrosis reported a clinical response in all patients [41]. One patient with cGVHD died from a GVHD flare following ruxolitinib discontinuation; the remaining patients were successfully treated with a low maintenance dose of ruxolitinib or slow tapering following response to treatment. Similar findings have been reported in adult patients with aGVHD or cGVHD [35,42-44] and pediatric patients with aGVHD [45]. These data suggest that ruxolitinib provides clinical benefit and durable responses in patients with SR GVHD; however, further data from large prospective clinical trials are required.

\section{REACH trials}

The REACH studies are prospective clinical trials designed to evaluate ruxolitinib in patients with SR aGVHD (REACH1 and REACH2) or CGVHD (REACH 3). REACH1 is a nonrandomized, single-cohort, Phase II clinical trial. REACH2 and REACH3 are randomized Phase III clinical trials. The REACH trials are anticipated to enroll 70 (REACH1), 308 (REACH2) and 324 (REACH3) patients $\geq 12$ years of age. Notably, in addition to evaluating clinical outcomes, all three REACH trials will collect samples for exploratory correlative analyses between potential biomarkers and patient outcomes.

\section{REACH1}

\section{Patients \& study design}

REACH1 (ClinicalTrials.gov identifier, NCT02953678) is an open-label, single-cohort, multicenter, Phase II clinical trial conducted in the USA to investigate ruxolitinib in combination with corticosteroids for the treatment of SR grade 2-4 aGVHD (Figure 1). Diagnosis of SR disease requires $\geq 1$ of the following: GVHD increasing in stage in any organ or developing in a new organ after 3 days of $\geq 2 \mathrm{mg} / \mathrm{kg}$ methylprednisolone or equivalent, GVHD that has not improved in stage in $\geq 1$ organ after 7 days of $\geq 2 \mathrm{mg} / \mathrm{kg}$ methylprednisolone or equivalent, 




Figure 1. REACH1 study design.

aGVHD: Acute graft-versus-host disease; BID: Twice daily; cGVHD: Chronic graft-versus-host disease; CNI: Calcineurin inhibitor; DOR: Duration of response; EOT: End of treatment; FFS: Failure-free survival; IS: Immunosuppressive medication; MAGIC: Mount Sinai Acute GVHD International Consortium; MR: Malignancy relapse/recurrence; NR: No response; NRM: Nonrelapse mortality; ORR: Overall response rate; OS: Overall survival; PD: Progressive disease; PK: Pharmacokinetics; SR: Steroid-refractory.

development of GVHD in a new organ after $\geq 1 \mathrm{mg} / \mathrm{kg}$ methylprednisolone or equivalent for skin GVHD or patients who progress during tapering before a 50\% decrease in corticosteroids is achieved. GVHD stage will be evaluated from screening through the end of treatment using the Mount Sinai Acute GVHD International Consortium guidelines [46]. Patients are excluded if they have had $>1$ allogeneic HSCT, $>1$ systemic treatment in addition to corticosteroids for aGVHD, cGVHD including overlap syndrome, splenectomy, active uncontrolled infection, severe organ failure, relapse of primary disease, corticosteroid treatment for indications other than GVHD within 7 days of enrollment, JAK inhibitor treatment after allogeneic HSCT or treatment with any other investigational agent, device or procedure within 21 days or five half-lives (whichever is longer) of enrollment.

Eligible patients receive ruxolitinib $5 \mathrm{mg}$ twice daily (BID), with increase to $10 \mathrm{mg}$ BID if hematologic parameters are stable and no treatment-related toxicities are recorded after the first 3 days of treatment. Patients receive corticosteroids at a starting dose of oral prednisone $2.5 \mathrm{mg} / \mathrm{kg} /$ day or intravenous methylprednisolone $2.0 \mathrm{mg} / \mathrm{kg} / \mathrm{day}$, with tapering as appropriate. Patients are administered combination treatment until treatment failure, unacceptable toxicity or death.

\section{Objectives}

The primary objective is to assess the efficacy of ruxolitinib in combination with corticosteroids. Secondary objectives are to assess additional response and longer-term efficacy outcomes in the study population, the incidence and severity of AEs and to evaluate the pharmacokinetics (PK) of ruxolitinib when administered in combination with corticosteroids. Exploratory objectives are to evaluate the incidence of secondary graft failure and aGVHD recurrences; discontinuation or use of corticosteroids; discontinuation of immunosuppressive medications; incidence of cGVHD; and changes in cytokine expression, T-cell subsets and biomarkers with treatment. 


\section{End points}

The primary study end point is ORR at day 28 , defined as the proportion of patients with a CR, very good PR or PR. Day 28 was chosen for the primary end point based on a previously identified correlation between day 28 response and long-term survival [47], making it a potentially effective surrogate study end point. The key secondary end point is 6 month duration of response (DOR), defined as the time from first response until GVHD progression or death, and will be assessed when all patients complete the day 180 study visit. Additional secondary end points include ORR at days 14, 56 and 100; 3 month DOR; NRM; relapse rate; relapse-related mortality rate; failure-free survival (FFS); and OS and PK measures. AEs will be assessed from the time of consent through 30-35 days after the end of treatment. Exploratory end points include the incidence rate of cGVHD; average and cumulative corticosteroid dose; the proportion of patients who discontinue immunosuppressive medications; the rate of secondary graft failure (i.e., $>95 \%$ recipient cells any time after engraftment with no signs of relapse, or retransplantation because of secondary neutropenia and/or thrombocytopenia within 2 months of HSCT); rate of aGVHD flares; and a biomarker analysis correlated with efficacy and safety outcomes.

\section{REACH2}

\section{Patients \& study design}

REACH2 (ClinicalTrials.gov identifier, NCT02913261) is a randomized, open-label, multi-center, Phase III clinical trial of ruxolitinib versus investigator choice best available therapy (BAT) in patients with SR grade 2-4 aGVHD after allogeneic HSCT (Figure 2). Study sites in Asia, Australia, Canada and the EU are recruiting patients. The criteria used for diagnosis of SR aGVHD staging are similar to those used in REACH1 (see above), with additional criterion of failure to taper steroids, defined as the requirement for an increase in corticosteroid dose to methylprednisolone $\geq 2 \mathrm{mg} / \mathrm{kg} /$ day (or equivalent prednisone dose $\geq 2.5 \mathrm{mg} / \mathrm{kg} /$ day) or failure to taper the methylprednisolone dose to $<0.5 \mathrm{mg} / \mathrm{kg} /$ day (or equivalent prednisone dose $<0.625 \mathrm{mg} / \mathrm{kg} /$ day) for a minimum of 7 days. Patients are excluded if they have had $>1$ systemic treatment for aGVHD other than corticosteroids and calcineurin inhibitors, presentation with cGVHD or GVHD overlap syndrome, active uncontrolled infection, severe organ failure, allogeneic HSCT failure within 6 months before enrollment, relapse of primary disease, corticosteroid treatment for indications other than GVHD within 7 days of enrollment, JAK inhibitor treatment after allogeneic HSCT and treatment with any other investigational agent within 30 days or five half-lives (whichever is longer) of enrollment.

Patients meeting the eligibility criteria for SR aGVHD are randomized 1:1 to receive ruxolitinib $10 \mathrm{mg}$ orally BID or BAT, and stratified by aGVHD grade. The planned treatment duration is approximately 6 months; patients responding to treatment will undergo tapering off ruxolitinib (50\% dose reduction every 2 months) beginning on day 56 once off systemic corticosteroids. Concomitant treatment with standard supportive care (e.g., anti-infective medications and transfusion support) and corticosteroids \pm calcineurin inhibitors is permitted; however, other systemic treatments for aGVHD are not allowed. Patients randomized to ruxolitinib who do not achieve the primary end point at day 28 discontinue study treatment; those assigned to BAT who do not reach the primary end point may be eligible for crossover to the ruxolitinib treatment group. This allowance is deemed ethically necessary because currently available data suggest that these patients could benefit from treatment with ruxolitinib [39].

\section{Objectives}

The primary objective is to compare the efficacy of ruxolitinib versus BAT in patients with grade 2-4 SR aGVHD. The key secondary objective is to compare the rate of durable ORR at day 56 between ruxolitinib and BAT. Additional secondary objectives include ORR at days 14 and 56, DOR, cumulative steroid dose until day 56, event-free survival, FFS, NRM, malignancy relapse/progression, incidence of cGVHD, assessment of the PK of ruxolitinib in SR aGVHD patients, exposure-response relationship of ruxolitinib in SR-aGVHD, patient-reported outcomes and safety. Exploratory objectives include determination of mutation and expression status at baseline; evaluation of the effect of ruxolitinib on cytokines, aGVHD biomarkers and immune cell subsets; exploration of cytokines and aGVHD biomarkers as pharmacodynamic markers for ruxolitinib activity; and the evaluation of resource utilization. 


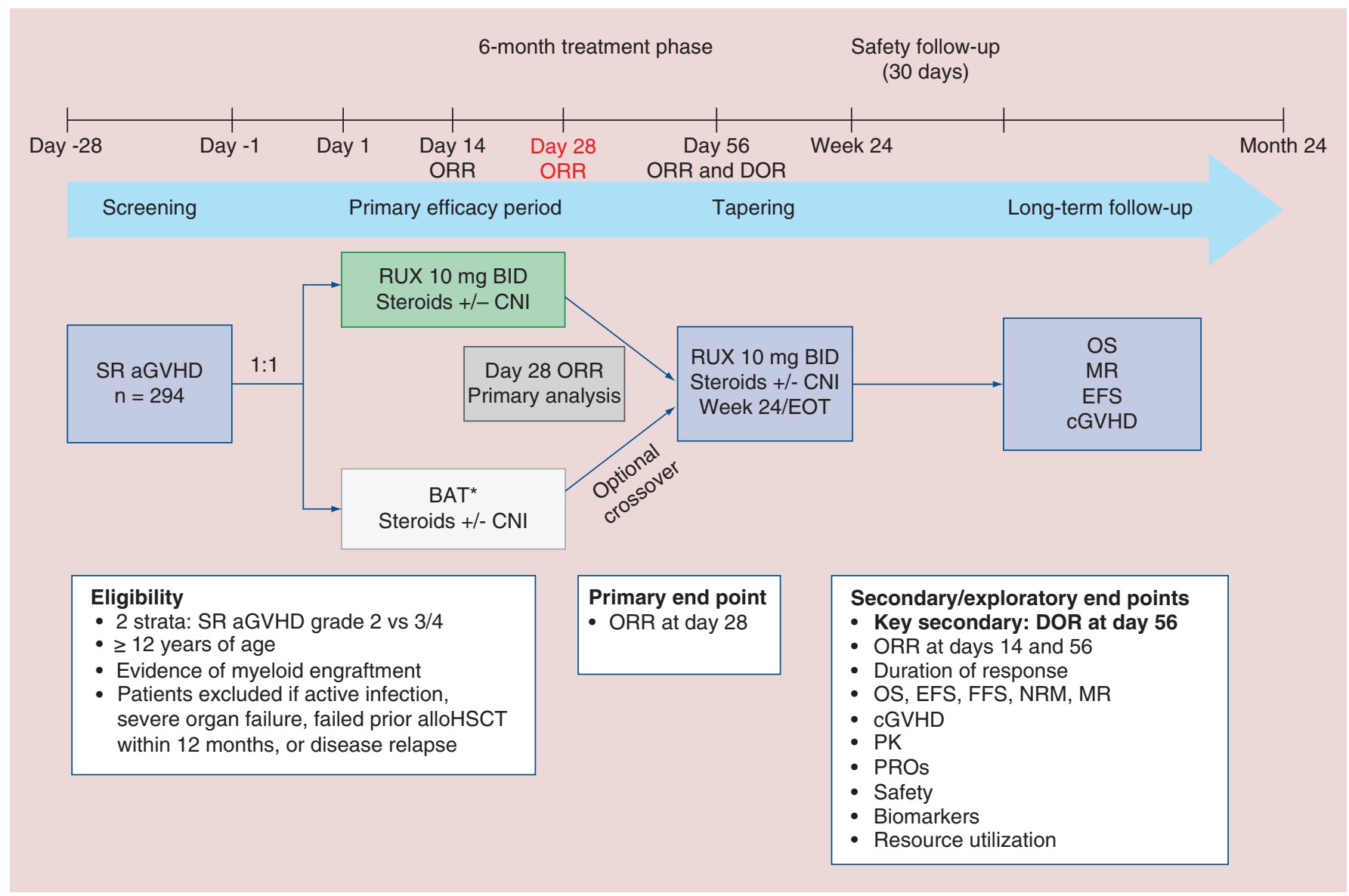

Figure 2. REACH2 study design.

*BAT is selected by the investigator prior to treatment randomization from the following standard systemic treatments: antithymocyte globulin, extracorporeal photopheresis, mesenchymal stromal cells, low-dose methotrexate, mycophenolate mofetil, mammalian target of rapamycin inhibitors (everolimus or sirolimus), etanercept or infliximab. No other BAT will be allowed.

aGVHD: Acute graft-versus-host disease; alloHSCT: Allogeneic hematopoietic stem cell transplant; BAT: Best available therapy; BID: Twice daily; CGVHD: Chronic graft-versus-host disease; CNI: Calcineurin inhibitor; DOR: Duration of response; EFS: Event-free survival; EOT: End of treatment; FFS: Failure-free survival; MR: Malignancy relapse/recurrence; NRM: Nonrelapse mortality; ORR: Overall response rate; OS: Overall survival; PK: Pharmacokinetics; PRO: Patient-reported outcome; RUX: Ruxolitinib; SR: Steroid-refractory.

\section{End points}

The primary end point of the study is ORR at day 28. The key secondary end point is the proportion of patients in each arm who achieve CR or PR at day 28 and maintain CR or PR at day 56. Other secondary end points include the proportion of patients who achieve ORR at days 14 and 56; DOR; and measures for determination of relapse, graft failure, overall mortality, NRM, diagnosis of cGVHD and single dose and steady state PK assessments. The exposure-response relationship will be evaluated by analysis of PK versus efficacy and safety. Patient-reported outcomes will be measured via changes in the Functional Assessment of Cancer Therapy-Bone Marrow Transplant (FACT-BMT) and EuroQol-5 Dimension (EQ-5D) instruments from baseline to each visit. Safety of study drug will be assessed by monitoring the frequency and severity of AEs. Exploratory end points include exploration of the relationship between mutational status, gene expression and response; changes in immune cell subsets, cytokines and aGVHD biomarkers; and relationship between subsets of soluble biomarkers over time with PK and efficacy. Resource utilization will be categorized based on time to discharge and the number and duration of hospital readmissions. 




Figure 3. REACH3 study design.

*BAT is selected by the investigator prior to treatment randomization from the following standard systemic treatments: extracorporeal photopheresis, low-dose methotrexate, mycophenolate mofetil, mammalian target of rapamycin inhibitors (everolimus or sirolimus), infliximab, rituximab, pentostatin or imatinib. Investigational agents only available via clinical trials (including JAK inhibitors or combinations of different treatments) are not permitted on the BAT arm. No other BAT will be allowed.

†The modified Lee Symptom Scale score is the key secondary end point for the US study centers only.

BAT: Best available therapy; BID: Twice daily; cGVHD: Chronic graft-versus-host disease; CNI: Calcineurin inhibitor; EOT: End of treatment; FFS: Failure-free survival; NRM: Nonrelapse mortality; ORR: Overall response rate; OS: Overall survival; PK; Pharmacokinetics; PRO: Patient-reported outcome; RUX: Ruxolitinib; SR: Steroid-refractory.

\section{REACH3}

\section{Patients \& study design}

REACH3 (ClinicalTrials.gov identifier, NCT03112603) is a randomized, open-label, multicenter, Phase III clinical trial assessing ruxolitinib versus investigator choice BAT in patients with SR cGVHD following allogeneic HSCT (Figure 3). The study will be conducted at several sites across Asia, the EU, the Middle East and North America. Diagnosis of SR cGVHD requires $\geq 1$ of the following: lack of response or cGVHD progression with $\geq 1 \mathrm{mg} / \mathrm{kg} / \mathrm{day}$ prednisone for $\geq 1$ week, cGVHD persistence without improvement following $>0.5 \mathrm{mg} / \mathrm{kg} /$ day or $1 \mathrm{mg} / \mathrm{kg}$ every other day for $\geq 4$ weeks or prednisone dose increased to $>0.25 \mathrm{mg} / \mathrm{kg} /$ day after two failed attempts at tapering the dose. Criteria for cGVHD staging are per the National Institutes of Health consensus criteria [8]. Patients are excluded if they transition from active aGVHD to cGVHD without tapering off corticosteroids ( \pm calcineurin inhibitor) and any systemic treatment, allogeneic HSCT failure within 6 months before enrollment, active uncontrolled infection or relapse of primary disease. Prior JAK inhibitor treatment for aGVHD is permitted for patients who achieved CR or PR and terminated treatment for $\geq 8$ weeks. 
Patients meeting the eligibility criteria for cGVHD will be randomized 1:1 to receive ruxolitinib $10 \mathrm{mg}$ orally BID or BAT per label and institutional guidelines and will be stratified by cGVHD severity (moderate vs severe). Patients may receive standard supportive care and cGVHD prophylaxis, such as corticosteroids and calcineurin inhibitors. Patients randomized to ruxolitinib who do not receive benefit per investigator judgment will discontinue study treatment. Patients in the BAT arm who do not achieve PR or better may cross over to ruxolitinib on or after cycle 7 day 1 . End of study occurs when all patients have completed 39 treatment cycles (each cycle is 4 weeks), discontinued from the study or died.

\section{Objectives}

The primary objective of the study is to compare the efficacy of ruxolitinib versus BAT in patients with moderate or severe SR cGVHD. The key secondary objectives are to compare the change in modified Lee Symptom Scale score (for study centers in the USA) and FFS (for all other participating study centers). Additional secondary objectives include assessment of best overall response, ORR at the end of cycle 3, DOR, OS, NRM, corticosteroid use, malignancy relapse/recurrence, changes in FACT-BMT and EQ-5D, assessment of PK of ruxolitinib in cGVHD patients, evaluation of safety and medical resource utilization. Exploratory objectives include determination of mutation and expression status at baseline; recurrence of malignant clones; effects on cytokines, cGVHD biomarkers and immune cell subsets; evaluation of ruxolitinib on markers of bone development in pediatric patients; and exploration of cGVHD recurrence following completion or tapering of systemic therapy.

\section{End points}

The primary study end point is ORR as defined by the 2014 National Institutes of Health consensus criteria [48] on cycle 7 day 1 (week 24). The key secondary end point is the proportion of patients with clinically relevant improvement in the modified Lee Symptom Scale score at cycle 7 day 1 (USA) or the composite time to event including the following FFS events: relapse/recurrence or death, NRM, or addition or initiation of another systemic therapy for cGVHD (regions outside of the USA). Other secondary end points include assessment of the proportion of patients achieving ORR at any time point and specifically at the end of cycle 3 (cycle 4 day 1); DOR, defined as the time from first response until cGVHD progression, death or the date of change/addition of systemic therapies for cGVHD; OS, NRM, malignancy relapse/recurrence; changes in FACT-BMT and EQ-5D; and PK measurements after a single dose and at steady state. Resource utilization will be evaluated by duration and frequency of hospitalization, emergency department and urgent care visits and additional outpatient visits. Exploratory end points include evaluating the relationship between mutational status and gene expression with response; mutational status over time; changes in immune cell subsets, cytokines and soluble cGVHD biomarkers; assessment of changes in soluble markers for bone resorption and formation; and evaluation of time to recurrence.

\section{Discussion}

Although a recent trend analysis suggested that incidences of aGVHD and cGVHD following allogeneic HSCT have decreased in recent years [49], GVHD continues to pose a significant burden to transplant success and represents a leading cause of NRM $[8,18,19]$. The three REACH trials comprise the largest and most comprehensive prospective clinical trials to date evaluating a JAK1/JAK2 inhibitor in GVHD settings. Results from REACH will play an important role in advancing how we treat patients with SR aGVHD and cGVHD.

The REACH study designs include several strengths. All three trials include patients who are $\geq 12$ years of age, permitting inclusion of adolescent patients. Based on an analysis by the Center for International Blood \& Marrow Transplant Research, more than 1000 patients/year $<18$ years of age received allogeneic transplants from URDs since 2009 [3], underscoring the importance of evaluating investigational treatments for GVHD in these patients. The Phase III clinical trials (REACH2 and REACH3) compare ruxolitinib with current preferred treatment options. Patients with aGVHD or cGVHD report negative effects on quality of life as a result of their disease. As such, it will be important for new treatment options to ameliorate the burdensome symptoms of GVHD in addition to treating the clinical manifestations to improve patient outcomes. Importantly, both Phase III REACH trials will evaluate measures of patient-reported quality of life. Finally, all three REACH trials include exploratory analyses to identify biomarkers that may correlate with patient outcomes. Biomarkers evaluated in these analyses may permit the identification of patients who will best respond to ruxolitinib treatment or optimal dosing strategies. The primary limitation of the REACH studies is the open-label design, which was deemed necessary for evaluation against investigator-preferred BAT as a comparator. 


\section{Conclusion \& future perspective}

Allogeneic HSCT offers a potentially curative treatment option for several hematologic malignancies and disorders [1-3]. However, despite evolving prophylactic care in the field of transplantation, GVHD continues to pose a substantial burden and represents a leading barrier to HSCT success [3,18]. Corticosteroids are the preferred first-line treatment option for patients with aGVHD or cGVHD, but approximately half of patients become refractory to steroids [20-23] and require second-line treatment.

Ruxolitinib is a JAK1/JAK2 inhibitor with the potential to treat GVHD in SR patients based on retrospective clinical data [39]. The three ongoing prospective REACH trials are currently enrolling patients to evaluate the therapeutic potential of ruxolitinib for GVHD and offer insight into GVHD disease pathophysiology.

It is clear that the pathogenesis of GVHD is biologically complex and leads to a heterogenous phenotype. With the advent of targeted therapies, we have a unique opportunity to further refine and iterate which subsets of patients with GVHD respond to a particular agent. Similar to antineoplastic therapy, it is very likely that GVHD will be treated with a combination of targeted therapies. The era of targeted therapy will allow us to refine biomarker validation (e.g., inflammatory cytokines, gene expression and regulatory T-cell induction) that can predict outcomes to a specific agent or within a specific subset of GVHD patients. Eventually, clinical trials will enroll unique subsets of patients with GVHD defined by the clinical characteristics, biomarker panels or evidence of certain pathways that are targetable by the proposed intervention. This will allow for a more 'personalized' approach, similar to the genomic-based approach used in oncology settings. The ultimate goal of GVHD therapy is not just to control the disease process, but to facilitate true immunotolerance that will allow patients to be successfully weaned off of therapy without residual GVHD side effects, while maintaining the beneficial GVL effect. Additionally, the role of therapeutic JAK inhibition as first-line or prophylactic treatment of GVHD, in addition to exploration of

\section{Executive summary}

\section{Background}

- Allogeneic hematopoietic stem cell transplantation (HSCT) is a potentially curative treatment for several hematologic disorders.

- Despite advances in HSCT methodology and supportive care, graft-versus-host disease (GVHD) develops in approximately $50 \%$ of HSCT recipients, and can manifest in acute (aGVHD) or chronic (cGVHD) forms.

- Corticosteroids serve as a first-line treatment option for acute and chronic GVHD, but many patients become steroid-refractory (SR). Ibrutinib is approved for the treatment of SR CGVHD, while there are no approved treatment options for SR aGVHD.

Overview of ruxolitinib \& rationale for targeting JAK signaling for SR GVHD

- The JAKs are intracellular nonreceptor tyrosine kinases predicted to be involved in the pathogenesis of GVHD.

- Ruxolitinib is a potent JAK1/JAK2 inhibitor shown to alleviate symptoms of GVHD in animal models as well as in patients with GVHD in several small studies and retrospective clinical reports.

\section{REACH trials}

- REACH1 is a single-arm, multicenter phase 2 study to investigate ruxolitinib in combination with corticosteroids in SR aGVHD (grades 2-4). The primary endpoint is ORR at Day 28 of treatment.

- REACH2 is an open-label, multicenter phase 3 study of crossover design to investigate ruxolitinib versus investigator's choice of best available therapy (BAT) in SR aGVHD (grades 2 to 4). The primary endpoint is ORR at Day 28 of treatment.

- REACH3 is an open-label, multicenter phase 3 study of crossover design to investigate ruxolitinib versus investigator's choice of BAT in SR CGVHD. The primary endpoint is ORR at Cycle 7, Day 1 (Week 24) of treatment.

Discussion

- GVHD following HSCT continues to pose a significant threat to transplant success and represents a leading cause of non-relapse mortality.

- The REACH trials are large and comprehensive prospective clinical trials of the JAK1/JAK2 inhibitor ruxolitinib in patients with SR aGVHD and CGVHD. Findings from these studies will inform future GVHD treatment strategies and may provide key insights into disease pathophysiology.

Future perspective

- The future of GVHD treatment likely lies in the development and refinement of targeted, personalized approaches.

- Pending positive results from the REACH trials, ruxolitinib may soon be integrated into aGVHD and cGVHD treatment regimens as a standard-of-care therapeutic. 
safety and efficacy of JAK1-specific (e.g., itacitinib) and JAK2-specific (e.g., pacritinib) inhibitors versus JAK1/2 inhibitors (e.g., ruxolitinib and baricitinib), may be explored in the coming years.

Further advancement of the field will require a well-coordinated, systematic, international effort with carefully annotated biological samples in the context of clinical trials, as well as a logical approach to drug development. We expect ruxolitinib will be integrated into this targeted approach as a standard-of-care therapy for SR aGVHD and cGVHD patients. In the context of cGVHD, ruxolitinib may be explored in combination with the recently approved Bruton tyrosine kinase inhibitor ibrutinib to enhance clinical benefit.

\section{Financial \& competing interests disclosure}

$M$ Jagasia has received research funding from Janssen and Mallinckrodt Pharmaceuticals. R Zeiser has received honoraria from Jazz Pharmaceuticals, Novartis and BMS. M Arbushites and P Delaite are employees of Incyte Corporation. B Gadbaw is an employee of Novartis. N von Bubnoff has received honoraria from Novartis and BMS and research funding from Novartis. The authors have no other relevant affiliations or financial involvement with any organization or entity with a financial interest in or financial conflict with the subject matter or materials discussed in the manuscript apart from those disclosed.

Editorial assistance was provided by Cory Pfeiffenberger, at Complete Healthcare Communications, LLC (West Chester, PA, USA), a CHC Group company, and funded by Incyte Corporation.

\section{Open access}

This work is licensed under the Attribution-NonCommercial-NoDerivatives 4.0 Unported License. To view a copy of this license, visit http://creativecommons.org/licenses/by-nc-nd/4.0/

\section{References}

Papers of special note have been highlighted as: $\bullet$ of interest; $\bullet \bullet$ of considerable interest

1 Magenau J, Runaas L, Reddy P. Advances in understanding the pathogenesis of graft-versus-host disease. Br. J. Haematol. 173(2), 190-205 (2016).

- This recent review provides a comprehensive overview of graft-versus-host disease (GVHD) pathophysiology, including the role of several immune cell subsets, and identifies pathways of interest for targeting with potential therapeutic agents.

2 Barriga F, Ramirez P, Wietstruck A, Rojas N. Hematopoietic stem cell transplantation: clinical use and perspectives. Biol. Res. 45(3), 307-316 (2012).

3 D'Souza A, Zhu X. Current uses and outcomes of hematopoietic cell transplantation (HCT): CIBMTR summary slides, 2016. www.cibmtr.org

4 Wynn R. Stem cell transplantation in inherited metabolic disorders. Hematology Am. Soc. Hematol. Educ. Program. 2011, 285-291 (2011).

5 Reddy P, Maeda Y, Liu C, Krijanovski OI, Korngold R, Ferrara JL. A crucial role for antigen-presenting cells and alloantigen expression in graft-versus-leukemia responses. Nat. Med. 11(11), 1244-1249 (2005).

6 Filipovich AH, Weisdorf D, Pavletic S et al. National Institutes of Health consensus development project on criteria for clinical trials in chronic graft-versus-host disease: I. Diagnosis and staging working group report. Biol. Blood Marrow Transplant. 11(12), 945-956 (2005).

7 Dignan FL, Clark A, Amrolia P et al. Diagnosis and management of acute graft-versus-host disease. Br. J. Haematol. 158(1), 30-45 (2012).

8 Jagasia MH, Greinix HT, Arora M et al. National Institutes of Health consensus development project on criteria for clinical trials in chronic graft-versus-host disease: I. the 2014 diagnosis and staging working group report. Biol. Blood Marrow Transplant. 21(3), 389-401 e381 (2015).

9 Choi J, Ziga ED, Ritchey J et al. IFN $\gamma$ R signaling mediates alloreactive T-cell trafficking and GVHD. Blood 120(19), 4093-4103 (2012).

$10 \mathrm{Ma} \mathrm{HH}$, Ziegler J, Li C et al. Sequential activation of inflammatory signaling pathways during graft-versus-host disease (GVHD): early role for STAT1 and STAT3. Cell. Immunol. 268(1), 37-46 (2011).

11 Jagasia M, Arora M, Flowers ME et al. Risk factors for acute GVHD and survival after hematopoietic cell transplantation. Blood 119(1), 296-307 (2012).

12 Alexander KA, Flynn R, Lineburg KE et al. CSF-1-dependant donor-derived macrophages mediate chronic graft-versus-host disease. J. Clin. Invest. 124(10), 4266-4280 (2014).

13 Sarantopoulos S, Stevenson KE, Kim HT et al. High levels of B-cell activating factor in patients with active chronic graft-versus-host disease. Clin. Cancer Res. 13(20), 6107-6114 (2007).

14 Lazaryan A, Weisdorf DJ, DeFor T et al. Risk factors for acute and chronic graft-versus-host disease after allogeneic hematopoietic cell transplantation with umbilical cord blood and matched sibling donors. Biol. Blood Marrow Transplant. 22(1), 134-140 (2016). 
- Reports analysis of risk factors including age, conditioning regimen, stem cell source and human leukocyte antigen disparity in the development of acute and chronic GVHD, and the impact of GVHD on overall survival.

15 Flowers ME, Inamoto Y, Carpenter PA et al. Comparative analysis of risk factors for acute graft-versus-host disease and for chronic graft-versus-host disease according to National Institutes of Health consensus criteria. Blood 117(11), 3214-3219 (2011).

16 Ferrara JL, Levine JE, Reddy P, Holler E. Graft-versus-host disease. Lancet 373(9674), 1550-1561 (2009).

17 Loiseau P, Busson M, Balere ML et al. HLA Association with hematopoietic stem cell transplantation outcome: the number of mismatches at HLA-A, -B, -C, -DRB1, or -DQB1 is strongly associated with overall survival. Biol. Blood Marrow Transplant. 13(8), 965-974 (2007).

18 Gratwohl A, Brand R, Frassoni F et al. Cause of death after allogeneic haematopoietic stem cell transplantation (HSCT) in early leukaemias: an EBMT analysis of lethal infectious complications and changes over calendar time. Bone Marrow Transplant. 36(9), 757-769 (2005).

19 Center for International Blood \& Marrow Transplant Research (CIBMTR). HCT trends and survival data. www.cibmtr.org/referencecenter/slidesreports/summaryslides/Pages/index.aspx

20 Martin PJ, Rizzo JD, Wingard JR et al. First- and second-line systemic treatment of acute graft-versus-host disease: recommendations of the American Society of Blood and Marrow Transplantation. Biol. Blood Marrow Transplant. 18(8), 1150-1163 (2012).

21 Ruutu T, Gratwohl A, de Witte T et al. Prophylaxis and treatment of GVHD: EBMT-ELN working group recommendations for a standardized practice. Bone Marrow Transplant. 49(2), 168-173 (2014).

- Describes recommendations from the European Group for Blood and Marrow Transplantation and the European LeukemiaNet working group for the prevention of GVHD, and first- and second-line GVHD treatments following allogeneic hematopoietic stem cell transplantation. The guidelines highlight that there is no standard second-line treatment for aGVHD or cGVHD and that patients should be encouraged to enroll in clinical trials when possible.

22 Dignan FL, Amrolia P, Clark A et al. Diagnosis and management of chronic graft-versus-host disease. Br. J. Haematol. 158(1), 46-61 (2012).

23 Garnett C, Apperley JF, Pavlu J. Treatment and management of graft-versus-host disease: improving response and survival. Ther. Adv. Hematol. 4(6), 366-378 (2013).

24 Arai S, Margolis J, Zahurak M, Anders V, Vogelsang GB. Poor outcome in steroid-refractory graft-versus-host disease with antithymocyte globulin treatment. Biol. Blood Marrow Transplant. 8(3), 155-160 (2002).

25 Miklos D, Cutler C, Arora M et al. Multicenter open-label phase 2 study of ibrutinib in chronic graft versus host disease (cGVHD) after failure of corticosteroids. Blood 128(22), LBA-3 (2016).

26 Schwartz DM, Bonelli M, Gadina M, O’Shea JJ. Type I/II cytokines, JAKs, and new strategies for treating autoimmune diseases. Nat. Rev. Rheumatol. 12(1), 25-36 (2016).

27 Betts BC, Abdel-Wahab O, Curran SA et al. Janus kinase-2 inhibition induces durable tolerance to alloantigen by human dendritic cell-stimulated T cells yet preserves immunity to recall antigen. Blood 118(19), 5330-5339 (2011).

28 Chen X, Das R, Komorowski R et al. Blockade of interleukin- 6 signaling augments regulatory T-cell reconstitution and attenuates the severity of graft-versus-host disease. Blood 114(4), 891-900 (2009).

29 Zorn E, Kim HT, Lee SJ et al. Reduced frequency of FOXP3+ CD4+CD25+ regulatory T cells in patients with chronic graft-versus-host disease. Blood 106(8), 2903-2911 (2005).

30 Jackson SW, Jacobs HM, Arkatkar T et al. B cell IFN-gamma receptor signaling promotes autoimmune germinal centers via cell-intrinsic induction of BCL-6. J. Exp. Med. 213(5), 733-750 (2016).

31 Quintás-Cardama A, Vaddi K, Liu P et al. Preclinical characterization of the selective JAK1/2 inhibitor INCB018424: therapeutic implications for the treatment of myeloproliferative neoplasms. Blood 115(15), 3109-3117 (2010).

32 JAKAFI ${ }^{\circledR}$ (ruxolitinib). Full prescribing information, Incyte Corporation, Wilmington, DE, USA, 2016. www.jakafi.com/pdf/prescribing-information.pdf

33 Heine A, Held SA, Daecke SN et al. The JAK-inhibitor ruxolitinib impairs dendritic cell function in vitro and in vivo. Blood 122(7), 1192-1202 (2013).

34 Stickel N, Hanke K, Marschner D et al. MicroRNA-146a reduces MHC-II expression via targeting JAK/STAT signaling in dendritic cells after stem cell transplantation. Leukemia 31(12), 2732-2741 (2017).

35 Spoerl S, Mathew NR, Bscheider M et al. Activity of therapeutic JAK 1/2 blockade in graft-versus-host disease. Blood 123(24), 3832-3842 (2014).

-. This proof-of-concept study in a murine model demonstrated that inhibition of JAK1/JAK2 signaling with ruxolitinib reduced symptoms of GVHD and increased the number of regulatory T cells. Additionally, 6/6 patients with steroid-refractory GVHD demonstrated a clinical response to ruxolitinib with reduction in GVHD symptoms and in levels of proinflammatory cytokines.

Takahashi S, Hashimoto D, Hayase E, Teshima T. Topical ruxolitinib protects LGR5+ stem cells in the hair follicle and ameliorates skin graft-versus-host disease. Biol. Blood Marrow Transplant. 22(3), S21-S22 (2016). 
37 Choi J, Cooper ML, Alahmari B et al. Pharmacologic blockade of JAK1/JAK2 reduces GvHD and preserves the graft-versus-leukemia effect. PLoS ONE 9(10), e109799 (2014).

38 Carniti C, Gimondi S, Vendramin A et al. Pharmacologic inhibition of JAK1/JAK2 signaling reduces experimental murine acute GVHD while preserving GVT effects. Clin. Cancer Res. 21(16), 3740-3749 (2015).

- Demonstrated that administration of ruxolitinib in a murine model of aGVHD resulted in reduced T-cell infiltration into target organs and amelioration of GVHD symptoms. Notably, ruxolitinib did not impair the graft-versus-tumor effect in this model.

39 Zeiser R, Burchert A, Lengerke $\mathrm{C}$ et al. Ruxolitinib in corticosteroid-refractory graft-versus-host disease after allogeneic stem cell transplantation: a multicenter survey. Leukemia 29(10), 2062-2068 (2015).

-. This retrospective, multicenter survey study of 95 patients with steroid-refractory acute or chronic GVHD who received ruxolitinib salvage therapy demonstrated that $>80 \%$ of patients demonstrated a complete or partial response to ruxolitinib. Ruxolitinib treatment also resulted in robust 6-month overall survival rates in these patients.

40 Zeiser R, Burchert A, Lengerke C et al. Long-term follow-up of patients with corticosteroid-refractory graft-versus-host disease treated with ruxolitinib. Blood (ASH Annual Meeting Abstracts) 128(22), abstract 4561 (2016).

41 Mori Y, Ikeda K, Inomata T et al. Ruxolitinib treatment for GvHD in patients with myelofibrosis. Bone Marrow Transplant. 51(12), 1584-1587 (2016).

42 Khoury HJ, Kota VK, Arellano M et al. Ruxolitinib as sparing agent for steroid-dependent chronic graft-versus-host disease (cGVHD). Biol. Blood Marrow Transplant. 23(3), S373.

43 Assouan D, Lebon D, Charbonnier A, Royer B, Marolleau JP, Gruson B. Ruxolitinib as a promising treatment for corticosteroid-refractory graft-versus-host disease. Br. J. Haematol. doi:10.1111/bjh.14679 (2017) (Epub ahead of print).

44 Maffini E, Giaccone L, Festuccia M et al. Ruxolitinib in steroid refractory graft-vs.-host disease: a case report. J. Hematol. Oncol. 9(1), 67 (2016).

45 Khandelwal P, Teusink-Cross A, Nelson AS et al. Ruxolitinib as salvage therapy in steroid refractory acute graft versus host disease in pediatric hematopoietic stem cell transplant patients. Biol. Blood Marrow Transplant. doi:10.1016/j.bbmt.2017.1003.1029 (2017) (Epub ahead of print).

46 Harris AC, Young R, Devine S et al. International, multicenter standardization of acute graft-versus-host disease clinical data collection: a report from the Mount Sinai Acute GVHD International Consortium. Biol. Blood Marrow Transplant. 22(1), 4-10 (2016).

47 Levine JE, Logan B, Wu J et al. Graft-versus-host disease treatment: predictors of survival. Biol. Blood Marrow Transplant. 16(12), 1693-1699 (2010).

48 Lee SJ, Wolff D, Kitko C et al. Measuring therapeutic response in chronic graft-versus-host disease. National Institutes of Health consensus development project on criteria for clinical trials in chronic graft-versus-host disease: IV. The 2014 Response Criteria Working Group report. Biol. Blood Marrow Transplant. 21(6), 984-999 (2015).

49 Tanaka Y, Kurosawa S, Tajima K et al. Analysis of non-relapse mortality and causes of death over 15 years following allogeneic hematopoietic stem cell transplantation. Bone Marrow Transplant. 51(4), 553-559 (2016). 